\title{
Weyl-Euler-Lagrange Equations of Motion on Flat Manifold
}

\author{
Zeki Kasap \\ Department of Elementary Education, Faculty of Education, Pamukkale University, Kinikli Campus, Denizli, Turkey \\ Correspondence should be addressed to Zeki Kasap; zekikasap@hotmail.com
}

Received 27 April 2015; Accepted 11 May 2015

Academic Editor: John D. Clayton

Copyright (C) 2015 Zeki Kasap. This is an open access article distributed under the Creative Commons Attribution License, which permits unrestricted use, distribution, and reproduction in any medium, provided the original work is properly cited.

\begin{abstract}
This paper deals with Weyl-Euler-Lagrange equations of motion on flat manifold. It is well known that a Riemannian manifold is said to be flat if its curvature is everywhere zero. Furthermore, a flat manifold is one Euclidean space in terms of distances. Weyl introduced a metric with a conformal transformation for unified theory in 1918. Classical mechanics is one of the major subfields of mechanics. Also, one way of solving problems in classical mechanics occurs with the help of the Euler-Lagrange equations. In this study, partial differential equations have been obtained for movement of objects in space and solutions of these equations have been generated by using the symbolic Algebra software. Additionally, the improvements, obtained in this study, will be presented.
\end{abstract}

\section{Introduction}

Euler-Lagrangian (analogues) mechanics are very important tools for differential geometry and analytical mechanics. They have a simple method to describe the model for mechanical systems. The models for mechanical systems are related. Studies in the literature about the Weyl manifolds are given as follows. Liu and Jun expand electronic origins, molecular dynamics simulations, computational nanomechanics, and multiscale modelling of materials fields [1]. Tekkoyun and Yayli examined generalized-quaternionic Kählerian analogue of Lagrangian and Hamiltonian mechanical systems [2]. The study given in [3] has the particular purpose to examine the discussion Weyl and Einstein had over Weyl's 1918 unified field theory for reasons such as the epistemological implications. Kasap and Tekkoyun investigated Lagrangian and Hamiltonian formalism for mechanical systems using para-/pseudo-Kähler manifolds, representing an interesting multidisciplinary field of research [4]. Kasap obtained the Weyl-Euler-Lagrange and the Weyl-Hamilton equations on $\mathbb{R}_{n}^{2 n}$ which is a model of tangent manifolds of constant $W$ sectional curvature [5]. Kapovich demonstrated an existence theorem for flat conformal structures on finite-sheeted coverings over a wide class of Haken manifolds [6]. Schwartz accepted asymptotically Riemannian manifolds with nonnegative scalar curvature [7]. Kulkarni identified some new examples of conformally flat manifolds [8]. Dotti and
Miatello intend to find out the real cohomology ring of low dimensional compact flat manifolds endowed with one of these special structures [9]. Szczepanski presented a list of sixdimensional Kähler manifolds and he submitted an example of eight-dimensional Kähler manifold with finite group [10]. Bartnik showed that the mass of an asymptotically flat $n$ manifold is a geometric invariant [11]. González considered complete, locally conformally flat metrics defined on a domain $\Omega \subset S^{n}$ [12]. Akbulut and Kalafat established infinite families of nonsimply connected locally conformally flat (LCF) 4-manifold realizing rich topological types [13]. Zhu suggested that it is to give a classification of complete locally conformally flat manifolds of nonnegative Ricci curvature [14]. Abood studied this tensor on general class almost Hermitian manifold by using a new methodology which is called an adjoint $G$-structure space [15]. K. Olszak and Z. Olszak proposed paraquaternionic analogy of these ideas applied to conformally flat almost pseudo-Kählerian as well as almost para-Kählerian manifolds [16]. Upadhyay studied bounding question for almost manifolds by looking at the equivalent description of them as infranil manifolds $\Gamma \backslash L \rtimes G / G$ [17].

\section{Preliminaries}

Definition 1. With respect to tangent space given any point $p \in M$, it has a tangent space $T_{p} M$ isometric to $\mathbb{R}^{n}$. If one has a metric (inner-product) in this space $\langle,\rangle_{p}: T_{p} M \times T_{p} M \mapsto$ 
$\mathbb{R}$ defined on every point $p \in M, M$ is called a Riemannian manifold.

Definition 2. A manifold with a Riemannian metric is a flat manifold such that it has zero curvature.

Definition 3. A differentiable manifold $M$ is said to be an almost complex manifold if there exists a linear map $J$ : $T M \rightarrow T M$ satisfying $J^{2}=-i d$ and $J$ is said to be an almost complex structure of $M$, where $i$ is the identity (unit) operator on $V$ such that $V$ is the vector space and $J^{2}=J \circ J$.

Theorem 4. The integrability of the almost complex structure implies a relation in the curvature. Let $\left\{x_{1}, y_{1}, x_{2}, y_{2}, x_{3}, y_{3}\right\}$ be coordinates on $\mathbb{R}^{6}$ with the standard flat metric:

$$
d s^{2}=\sum_{i=1}^{3}\left(d x_{i}^{2}+d y_{i}^{2}\right)
$$

(see [18]).

Definition 5. A (pseudo-)Riemannian manifold is conformally flat manifold if each point has a neighborhood that can be mapped to flat space by a conformal transformation. Let $(M, g)$ be a pseudo-Riemannian manifold.

Theorem 6. Let $(M, g)$ be conformally flat if, for each point $x$ in $M$, there exists a neighborhood $U$ of $x$ and a smooth function $f$ defined on $U$ such that $\left(U, e^{2 f} g\right)$ is flat. The function $f$ need not be defined on all of $M$. Some authors use locally conformally flat to describe the above notion and reserve conformally flat for the case in which the function $f$ is defined on all of $M$ [19].

Definition 7. A pseudo-J-holomorphic curve is a smooth map from a Riemannian surface into an almost complex manifold such that it satisfies the Cauchy-Riemann equation [20].

Definition 8. A conformal map is a function which preserves angles as the most common case where the function is between domains in the complex plane. Conformal maps can be defined between domains in higher dimensional Euclidean spaces and more generally on a (semi-)Riemannian manifold.

Definition 9. Conformal geometry is the study of the set of angle-preserving (conformal) transformations on a space. In two real dimensions, conformal geometry is precisely the geometry of Riemannian surfaces. In more than two dimensions, conformal geometry may refer either to the study of conformal transformations of flat spaces (such as Euclidean spaces or spheres) or to the study of conformal manifolds which are Riemannian or pseudo-Riemannian manifolds with a class of metrics defined up to scale.

Definition 10. A conformal manifold is a differentiable manifold equipped with an equivalence class of (pseudo-)Riemann metric tensors, in which two metrics $g^{\prime}$ and $g$ are equivalent if and only if

$$
g^{\prime}=\Psi^{2} g
$$

where $\Psi>0$ is a smooth positive function. An equivalence class of such metrics is known as a conformal metric or conformal class and a manifold with a conformal structure is called a conformal manifold [21].

\section{Weyl Geometry}

Conformal transformation for use in curved lengths has been revealed. The linear distance between two points can be found easily by Riemann metric. Many scientists have used the Riemann metric. Einstein was one of the first to study this field. Einstein discovered the Riemannian geometry and successfully used it to describe general relativity in the 1910 that is actually a classical theory for gravitation. But the universe is really completely not like Riemannian geometry. Each path between two points is not always linear. Also, orbits of moving objects may change during movement. So, each two points in space may not be linear geodesic. Then, a method is required for converting nonlinear distance to linear distance. Weyl introduced a metric with a conformal transformation in 1918. The basic concepts related to the topic are listed below [22-24].

Definition 11. Two Riemann metrics $g_{1}$ and $g_{2}$ on $M$ are said to be conformally equivalent iff there exists a smooth function $f: M \rightarrow \mathbb{R}$ with

$$
e^{f} g_{1}=g_{2} \text {. }
$$

In this case, $g_{1} \sim g_{2}$.

Definition 12. Let $M$ be an $n$-dimensional smooth manifold. A pair $(M, G)$, where a conformal structure on $M$ is an equivalence class $G$ of Riemann metrics on $M$, is called a conformal structure.

Theorem 13. Let $\nabla$ be a connection on $M$ and $g \in G$ a fixed metric. $\nabla$ is compatible with $(M, G) \Leftrightarrow$; there exists a 1 -form $\omega$ with $\nabla_{X} g+\omega(X) g=0$.

Definition 14. A compatible torsion-free connection is called a Weyl connection. The triple $(M, G, \nabla)$ is a Weyl structure.

Theorem 15. To each metric $g \in G$ and 1 -form $\omega$, there corresponds a unique Weyl connection $\nabla$ satisfying $\nabla_{X} g+\omega(X) g=$ 0.

Definition 16. Define a function $F:\{1$-forms on $M\} \times G \rightarrow$ \{Weyl connections\} by $F(g, \omega)=\nabla$, where $\nabla$ is the connection guaranteed by Theorem 6 . One says that $\nabla$ corresponds to $(g, \omega)$.

Proposition 17. (1) F is surjective.

Proof. $F$ is surjective by Theorem 13.

(2) $F(g, \omega)=F\left(e^{f} g, \eta\right)$ iff $\eta=\omega-d f$. So

$$
F\left(e^{f} g\right)=F(g)-d f
$$

where $G$ is a conformal structure. Note that a Riemann metric $g$ and $a$ one-form $\omega$ determine a Weyl structure; namely, $F$ : 
$G \rightarrow \wedge^{1} M$, where $G$ is the equivalence class of $g$ and $F\left(e^{f} g\right)=$ $\omega-d f$.

Proof. Suppose that $F(g, \omega)=F\left(e^{f} g, \eta\right)=\nabla$. We have

$$
\begin{aligned}
\nabla_{X} & \left(e^{f} g\right)+\eta(X) e^{f} g \\
= & X\left(e^{f}\right) g+e^{f} \nabla_{X} g+\eta(X) e^{f} g \\
& =d f(X) e^{f} g+e^{f} \nabla_{X} g+\eta(X) e^{f} g=0 .
\end{aligned}
$$

Therefore, $\nabla_{X} g=-(d f(X)+\eta(X))$. On the other hand, $\nabla_{X} g+$ $\omega(X) g=0$. Therefore, $\omega=\eta+d f$. Set $\nabla=F(g, \omega)$. To show, $\nabla=F\left(e^{f} g, \eta\right)$ and $\nabla_{X}\left(e^{f} g\right)+\eta(X) e^{f} g=0$. To calculate

$$
\begin{aligned}
\nabla_{X} & \left(e^{f} g\right)+\eta(X) e^{f} g \\
& =e^{f} d f(X) g+e^{f} \nabla_{X} g+(\omega(X)-d f(X)) e^{f} g \\
& =e^{f}\left(\nabla_{X} g+\omega(X) g\right)=0 .
\end{aligned}
$$

Theorem 18. A connection on the metric bundle $\omega$ of a conformal manifold $M$ naturally induces a map $F: G \rightarrow \wedge^{1} M$ and (4) and conversely. Parallel translation of points in $\omega$ by the connection is the same as their translation by F.

Theorem 19. Let $\nabla$ be a torsion-free connection on the tangent bundle of $M$ and $m \geq 6$. If $(M, g, \nabla, J)$ is a Kähler-Weyl structure, then the associated Weyl structure is trivial; that is, there is a conformally equivalent metric $\widetilde{g}=e^{2 f} g$ so that $(M, \tilde{g}, J)$ is Kähler and so that $\nabla=\nabla^{\tilde{g}}[25-27]$.

Definition 20. Weyl curvature tensor is a measure of the curvature of spacetime or a pseudo-Riemannian manifold. Like the Riemannian curvature tensor, the Weyl tensor expresses the tidal force that a body feels when moving along a geodesic.

Definition 21. Weyl transformation is a local rescaling of the metric tensor: $g_{a b}(x) \rightarrow e^{-2 \omega(x)} g_{a b}(x)$ which produces another metric in the same conformal class. A theory or an expression invariant under this transformation is called conformally invariant, or is said to possess Weyl symmetry. The Weyl symmetry is an important symmetry in conformal field theory.

\section{Complex Structures on Conformally Flat Manifold}

In this section, Weyl structures on flat manifolds will be transferred to the mechanical system. Thus, the time-dependent Euler-Lagrange partial equations of motion of the dynamic system will be found. A flat manifold is something that locally looks like Euclidean space in terms of distances and angles. The basic example is Euclidean space with the usual metric $d s^{2}=\sum_{i} d x_{i}^{2}$. Any point on a flat manifold has a neighborhood isometric to a neighborhood in Euclidean space. A flat manifold is locally Euclidean in terms of distances and angles and merely topologically locally Euclidean, as all manifolds are. The simplest nontrivial examples occur as surfaces in four-dimensional space as the flat torus is a flat manifold. It is the image of $f(x, y)=(\cos x, \sin x, \cos y, \sin y)$.

Example 22. It vanishes if and only if $J$ is an integrable almost complex structure; that is, given any point $P \in M$, there exist local coordinates $\left(x_{i}, y_{i}\right), i=1,2,3$, centered at $P$, following structures taken from

$$
\begin{aligned}
& J \partial x_{1}=\cos \left(x_{3}\right) \partial y_{1}+\sin \left(x_{3}\right) \partial y_{2}, \\
& J \partial x_{2}=-\sin \left(x_{3}\right) \partial y_{1}+\cos \left(x_{3}\right) \partial y_{2}, \\
& J \partial x_{3}=\partial y_{3}, \\
& J \partial y_{1}=-\cos \left(x_{3}\right) \partial x_{1}+\sin \left(x_{3}\right) \partial x_{2}, \\
& J \partial y_{2}=-\sin \left(x_{3}\right) \partial x_{1}-\cos \left(x_{3}\right) \partial x_{2}, \\
& J \partial y_{3}=-\partial x_{3} .
\end{aligned}
$$

The above structures (7) have been taken from [28]. We will use $\partial x_{i}=\partial / \partial x_{i}$ and $\partial y_{i}=\partial / \partial y_{i}$.

The Weyl tensor differs from the Riemannian curvature tensor in that it does not convey information on how the volume of the body changes. In dimensions 2 and 3 the Weyl curvature tensor vanishes identically. Also, the Weyl curvature is generally nonzero for dimensions $\geq 4$. If the Weyl tensor vanishes in dimension $\geq 4$, then the metric is locally conformally flat: there exists a local coordinate system in which the metric tensor is proportional to a constant tensor. This fact was a key component for gravitation and general relativity [29].

Proposition 23. If we extend (7) by means of conformal structure [19, 30], Theorem 19 and Definition 21, we can give equations as follows:

$$
\begin{aligned}
& J \frac{\partial}{\partial x_{1}}=e^{2 f} \cos \left(x_{3}\right) \frac{\partial}{\partial y_{1}}+e^{2 f} \sin \left(x_{3}\right) \frac{\partial}{\partial y_{2}} \\
& J \frac{\partial}{\partial x_{2}}=-e^{2 f} \sin \left(x_{3}\right) \frac{\partial}{\partial y_{1}}+e^{2 f} \cos \left(x_{3}\right) \frac{\partial}{\partial y_{2}} \\
& J \frac{\partial}{\partial x_{3}}=e^{2 f} \frac{\partial}{\partial y_{3}} \\
& J \frac{\partial}{\partial y_{1}}=-e^{-2 f} \cos \left(x_{3}\right) \frac{\partial}{\partial x_{1}}+e^{-2 f} \sin \left(x_{3}\right) \frac{\partial}{\partial x_{2}} \\
& J \frac{\partial}{\partial y_{2}}=-e^{-2 f} \sin \left(x_{3}\right) \frac{\partial}{\partial x_{1}}-e^{-2 f} \cos \left(x_{3}\right) \frac{\partial}{\partial x_{2}} \\
& J \frac{\partial}{\partial y_{3}}=-e^{-2 f} \frac{\partial}{\partial x_{3}},
\end{aligned}
$$

such that they are base structures for Weyl-Euler-Lagrange equations, where $J$ is a conformal complex structure to be similar to an integrable almost complex J given in (7). From now on, we continue our studies thinking of the $(T M, g, \nabla, J)$ instead of 
Weyl manifolds (TM, g, $\nabla, J)$. Now, J denotes the structure of the holomorphic property:

$$
\begin{aligned}
& J^{2} \frac{\partial}{\partial x_{1}}=J \circ J \frac{\partial}{\partial x_{1}}=e^{2 f} \cos \left(x_{3}\right) J \frac{\partial}{\partial y_{1}}+e^{2 f} \sin \left(x_{3}\right) J \\
& \cdot \frac{\partial}{\partial y_{2}}=e^{2 f} \cos \left(x_{3}\right) \\
& \cdot\left[-e^{-2 f} \cos \left(x_{3}\right) \frac{\partial}{\partial x_{1}}+e^{-2 f} \sin \left(x_{3}\right) \frac{\partial}{\partial x_{2}}\right]+e^{2 f} \\
& \cdot \sin \left(x_{3}\right)\left[-e^{-2 f} \sin \left(x_{3}\right) \frac{\partial}{\partial x_{1}}-e^{-2 f} \cos \left(x_{3}\right) \frac{\partial}{\partial x_{2}}\right] \\
& =-\cos ^{2}\left(x_{3}\right) \frac{\partial}{\partial x_{1}}+\cos \left(x_{3}\right) \sin \left(x_{3}\right) \frac{\partial}{\partial x_{2}} \\
& -\sin ^{2}\left(x_{3}\right) \frac{\partial}{\partial x_{1}}-\sin _{3}\left(x_{3}\right) \cos \left(x_{3}\right) \frac{\partial}{\partial x_{2}} \\
& =-\left[\cos ^{2}\left(x_{3}\right)+\sin ^{2}\left(x_{3}\right)\right] \frac{\partial}{\partial x_{1}}=-\frac{\partial}{\partial x_{1}}
\end{aligned}
$$

and in similar manner it is shown that

$$
\begin{gathered}
J^{2} \frac{\partial}{\partial x_{i}}=-\frac{\partial}{\partial x_{i}}, \\
J^{2} \frac{\partial}{\partial y_{i}}=-\frac{\partial}{\partial y_{i}}
\end{gathered}
$$

$$
i=1,2,3 \text {. }
$$

As can be seen from (9) and (10) $J^{2}=-I$ are the complex structures.

\section{Euler-Lagrange Dynamics Equations}

Definition 24 (see [31-33]). Let $M$ be an $n$-dimensional manifold and $T M$ its tangent bundle with canonical projection $\tau_{M}: T M \rightarrow M . T M$ is called the phase space of velocities of the base manifold $M$. Let $L: T M \rightarrow \mathbb{R}$ be a differentiable function on $T M$ and it is called the Lagrangian function. We consider closed 2-form on $T M$ and $\Phi_{L}=-d \mathbf{d}_{J} L$. Consider the equation

$$
\mathbf{i}_{V} \Phi_{L}=d E_{L}
$$

where the semispray $V$ is a vector field. Also, $\mathbf{i}$ is a reducing function and $\mathbf{i}_{V} \Phi_{L}=\Phi_{L}(V)$. We will see that, for motion in a potential, $E_{L}=\mathbf{V}(L)-L$ is an energy function $(L=T-P=$ $(1 / 2) m v^{2}-m g h$, kinetic-potential energies) and $\mathbf{V}=J V$ a Liouville vector field. Here, $d E_{L}$ denotes the differential of $E$. We will see that (11) under a certain condition on $V$ is the intrinsic expression of the Euler-Lagrange equations of motion. This equation is named Euler-Lagrange dynamical equation. The triple $\left(T M, \Phi_{L}, V\right)$ is known as EulerLagrangian system on the tangent bundle TM. The operations run on (11) for any coordinate system $\left(q^{i}(t), p_{i}(t)\right)$. Infinite dimension Lagrangian's equation is obtained in the form below:

$$
\begin{aligned}
\frac{d}{d t}\left(\frac{\partial L}{\partial \dot{q}^{i}}\right)-\frac{\partial L}{\partial q^{i}} & =0 \\
\frac{d q^{i}}{d t} & =\dot{q}^{i}
\end{aligned}
$$

$$
i=1, \ldots, n \text {. }
$$

\section{Conformal Weyl-Euler-Lagrangian Equations}

Here, we, using (11), obtain Weyl-Euler-Lagrange equations for classical and quantum mechanics on conformally flat manifold and it is shown by $(T M, g, \nabla, J)$.

Proposition 25. Let $\left(x_{i}, y_{i}\right)$ be coordinate functions. Also, on $(T M, g, \nabla, J)$, let $V$ be the vector field determined by $V=$ $\sum_{i=1}^{3}\left(X^{i}\left(\partial / \partial x_{i}\right)+Y^{i}\left(\partial / \partial y_{i}\right)\right)$. Then the vector field defined by

$$
\begin{aligned}
\mathbf{V}= & J V \\
= & X^{1}\left(e^{2 f} \cos \left(x_{3}\right) \frac{\partial}{\partial y_{1}}+e^{2 f} \sin \left(x_{3}\right) \frac{\partial}{\partial y_{2}}\right) \\
& +X^{2}\left(-e^{2 f} \sin \left(x_{3}\right) \frac{\partial}{\partial y_{1}}+e^{2 f} \cos \left(x_{3}\right) \frac{\partial}{\partial y_{2}}\right) \\
& +X^{3} e^{2 f} \frac{\partial}{\partial y_{3}} \\
& +Y^{1}\left(-e^{-2 f} \cos \left(x_{3}\right) \frac{\partial}{\partial x_{1}}+e^{-2 f} \sin \left(x_{3}\right) \frac{\partial}{\partial x_{2}}\right) \\
& +Y^{2}\left(-e^{-2 f} \sin \left(x_{3}\right) \frac{\partial}{\partial x_{1}}-e^{-2 f} \cos \left(x_{3}\right) \frac{\partial}{\partial x_{2}}\right) \\
& -Y^{3} e^{-2 f} \frac{\partial}{\partial x_{3}}
\end{aligned}
$$

is thought to be Weyl-Liouville vector field on conformally flat manifold $(T M, g, \nabla, J) . \Phi_{L}=-d \mathbf{d}_{J} L$ is the closed 2-form given by (11) such that $\mathbf{d}=\sum_{i=1}^{3}\left(\left(\partial / \partial x_{i}\right) d x_{i}+\left(\partial / \partial y_{i}\right) d y_{i}\right)$, $\mathbf{d}_{J}: F(M) \rightarrow \wedge^{1} M, \mathbf{d}_{J}=i_{J} \mathbf{d}-\mathbf{d} i_{J}$, and $\mathbf{d}_{J}=J(\mathbf{d})=$ $\sum_{i=1}^{3}\left(X^{i} J\left(\partial / \partial x_{i}\right)+Y^{i} J\left(\partial / \partial y_{i}\right)\right)$. Also, the vertical differentiation $\mathbf{d}_{J}$ is given where $d$ is the usual exterior derivation. Then, there is the following result. We can obtain Weyl-Euler-Lagrange equations for classical and quantum mechanics on conformally flat manifold (TM, $g, \nabla, J)$. We get the equations given by

$$
\begin{aligned}
\mathbf{d}_{J}= & {\left[e^{2 f} \cos \left(x_{3}\right) \frac{\partial}{\partial y_{1}}+e^{2 f} \sin \left(x_{3}\right) \frac{\partial}{\partial y_{2}}\right] d x_{1} } \\
& +\left[-e^{2 f} \sin \left(x_{3}\right) \frac{\partial}{\partial y_{1}}+e^{2 f} \cos \left(x_{3}\right) \frac{\partial}{\partial y_{2}}\right] d x_{2} \\
& +e^{2 f} \frac{\partial}{\partial y_{3}} d x_{3}
\end{aligned}
$$




$$
\begin{aligned}
& +\left[-e^{-2 f} \cos \left(x_{3}\right) \frac{\partial}{\partial x_{1}}+e^{-2 f} \sin \left(x_{3}\right) \frac{\partial}{\partial x_{2}}\right] d y_{1} \\
& +\left[-e^{-2 f} \sin \left(x_{3}\right) \frac{\partial}{\partial x_{1}}-e^{-2 f} \cos \left(x_{3}\right) \frac{\partial}{\partial x_{2}}\right] d y_{2} \\
& -e^{-2 f} \frac{\partial}{\partial x_{3}} d y_{3} .
\end{aligned}
$$

Also,

$$
\begin{aligned}
\Phi_{L} & =-d \mathbf{d}_{J} L \\
& =-d\left(\left[e^{2 f} \cos \left(x_{3}\right) \frac{\partial}{\partial y_{1}}+e^{2 f} \sin \left(x_{3}\right) \frac{\partial}{\partial y_{2}}\right] d x_{1}\right. \\
& +\left[-e^{2 f} \sin \left(x_{3}\right) \frac{\partial}{\partial y_{1}}+e^{2 f} \cos \left(x_{3}\right) \frac{\partial}{\partial y_{2}}\right] d x_{2} \\
& +e^{2 f} \frac{\partial L}{\partial y_{3}} d x_{3} \\
& +\left[-e^{-2 f} \cos \left(x_{3}\right) \frac{\partial}{\partial x_{1}}+e^{-2 f} \sin \left(x_{3}\right) \frac{\partial}{\partial x_{2}}\right] d y_{1} \\
& +\left[-e^{-2 f} \sin \left(x_{3}\right) \frac{\partial}{\partial x_{1}}-e^{-2 f} \cos \left(x_{3}\right) \frac{\partial}{\partial x_{2}}\right] d y_{2} \\
& \left.-e^{-2 f} \frac{\partial L}{\partial x_{3}} d y_{3}\right)
\end{aligned}
$$

and then we find

$$
\mathbf{i}_{V} \Phi_{L}=\Phi_{L}(V)=\Phi_{L}\left(\sum_{i=1}^{3}\left(X^{i} \frac{\partial}{\partial x_{i}}+Y^{i} \frac{\partial}{\partial y_{i}}\right)\right) .
$$

Moreover, the energy function of system is

$$
\begin{aligned}
E_{L}= & X^{1}\left[e^{2 f} \cos \left(x_{3}\right) \frac{\partial L}{\partial y_{1}}+e^{2 f} \sin \left(x_{3}\right) \frac{\partial L}{\partial y_{2}}\right] \\
& +X^{2}\left[-e^{2 f} \sin \left(x_{3}\right) \frac{\partial L}{\partial y_{1}}+e^{2 f} \cos \left(x_{3}\right) \frac{\partial L}{\partial y_{2}}\right] \\
& +X^{3} e^{2 f} \frac{\partial L}{\partial y_{3}} \\
& +Y^{1}\left[-e^{-2 f} \cos \left(x_{3}\right) \frac{\partial L}{\partial x_{1}}+e^{-2 f} \sin \left(x_{3}\right) \frac{\partial L}{\partial x_{2}}\right] \\
& +Y^{2}\left[-e^{-2 f} \sin \left(x_{3}\right) \frac{\partial L}{\partial x_{1}}-e^{-2 f} \cos \left(x_{3}\right) \frac{\partial L}{\partial x_{2}}\right] \\
& -Y^{3} e^{-2 f} \frac{\partial L}{\partial x_{3}}-L
\end{aligned}
$$

and the differential of $E_{L}$ is

$$
\begin{gathered}
d E_{L}=X^{1}\left(e^{2 f} \cos \left(x_{3}\right) \frac{\partial^{2} L}{\partial x_{1} \partial y_{1}} d x_{1}\right. \\
+2 e^{2 f} \cos \left(x_{3}\right) \frac{\partial f}{\partial x_{1}} \frac{\partial L}{\partial y_{1}} d x_{1} \\
+e^{2 f} \sin \left(x_{3}\right) \frac{\partial^{2} L}{\partial x_{1} \partial y_{2}} d x_{1}
\end{gathered}
$$$$
\left.+2 e^{2 f} \sin \left(x_{3}\right) \frac{\partial f}{\partial x_{1}} \frac{\partial L}{\partial y_{2}} d x_{1}\right)
$$$$
+X^{2}\left(-e^{2 f} \sin \left(x_{3}\right) \frac{\partial^{2} L}{\partial x_{1} \partial y_{1}} d x_{1}\right.
$$$$
-2 e^{2 f} \sin \left(x_{3}\right) \frac{\partial f}{\partial x_{1}} \frac{\partial L}{\partial y_{1}} d x_{1}
$$$$
+e^{2 f} \cos \left(x_{3}\right) \frac{\partial^{2} L}{\partial x_{1} \partial y_{2}} d x_{1}
$$$$
\left.+2 e^{2 f} \cos \left(x_{3}\right) \frac{\partial f}{\partial x_{1}} \frac{\partial L}{\partial y_{2}} d x_{1}\right)
$$$$
+X^{3}\left(e^{2 f} \frac{\partial^{2} L}{\partial x_{1} \partial y_{3}} d x_{1}+2 e^{2 f} \frac{\partial f}{\partial x_{1}} \frac{\partial L}{\partial y_{3}} d x_{1}\right)
$$$$
+Y^{1}\left(-e^{-2 f} \cos \left(x_{3}\right) \frac{\partial^{2} L}{\partial x_{1}^{2}} d x_{1}\right.
$$$$
+2 e^{-2 f} \cos \left(x_{3}\right) \frac{\partial f}{\partial x_{1}} \frac{\partial L}{\partial x_{1}} d x_{1}
$$$$
+e^{-2 f} \sin \left(x_{3}\right) \frac{\partial^{2} L}{\partial x_{1} \partial x_{2}} d x_{1}
$$$$
\left.-2 e^{-2 f} \sin \left(x_{3}\right) \frac{\partial f}{\partial x_{1}} \frac{\partial L}{\partial x_{2}} d x_{1}\right)
$$$$
+Y^{2}\left(-e^{-2 f} \sin \left(x_{3}\right) \frac{\partial^{2} L}{\partial x_{1}^{2}} d x_{1}\right.
$$$$
+2 e^{-2 f} \sin \left(x_{3}\right) \frac{\partial f}{\partial x_{1}} \frac{\partial L}{\partial x_{1}} d x_{1}
$$$$
-e^{-2 f} \cos \left(x_{3}\right) \frac{\partial^{2} L}{\partial x_{1} \partial x_{2}} d x_{1}
$$

$$
\begin{aligned}
& \left.+2 e^{-2 f} \cos \left(x_{3}\right) \frac{\partial f}{\partial x_{1}} \frac{\partial L}{\partial x_{2}} d x_{1}\right) \\
& +Y^{3}\left(-\frac{\partial^{2} L}{\partial x_{1} \partial x_{3}} d x_{1}+2 e^{-2 f} \frac{\partial f}{\partial x_{1}} \frac{\partial}{\partial x_{3}} d x_{1}\right) \\
& -\frac{\partial L}{\partial x_{1}} d x_{1}+X^{1}\left(e^{2 f} \cos \left(x_{3}\right) \frac{\partial^{2} L}{\partial x_{2} \partial y_{1}} d x_{2}\right.
\end{aligned}
$$




$$
\begin{aligned}
& +2 e^{2 f} \cos \left(x_{3}\right) \frac{\partial f}{\partial x_{2}} \frac{\partial L}{\partial y_{1}} d x_{2} \\
& +2 e^{2 f} \sin \left(x_{3}\right) \frac{\partial f}{\partial x_{3}} \frac{\partial L}{\partial y_{2}} d x_{3} \\
& +e^{2 f} \sin \left(x_{3}\right) \frac{\partial^{2} L}{\partial x_{2} \partial y_{2}} d x_{2} \\
& \left.+2 e^{2 f} \sin \left(x_{3}\right) \frac{\partial f}{\partial x_{2}} \frac{\partial L}{\partial y_{2}} d x_{2}\right) \\
& +X^{2}\left(-e^{2 f} \sin \left(x_{3}\right) \frac{\partial^{2} L}{\partial x_{2} \partial y_{1}} d x_{2}\right. \\
& -2 e^{2 f} \sin \left(x_{3}\right) \frac{\partial f}{\partial x_{2}} \frac{\partial L}{\partial y_{1}} d x_{2} \\
& +e^{2 f} \cos \left(x_{3}\right) \frac{\partial^{2} L}{\partial x_{2} \partial y_{2}} d x_{2} \\
& \left.+2 e^{2 f} \cos \left(x_{3}\right) \frac{\partial f}{\partial x_{2}} \frac{\partial L}{\partial y_{2}} d x_{2}\right) \\
& +X^{3}\left(e^{2 f} \frac{\partial^{2} L}{\partial x_{2} \partial y_{3}} d x_{2}+2 e^{2 f} \frac{\partial f}{\partial x_{2}} \frac{\partial L}{\partial y_{3}} d x_{2}\right) \\
& +Y^{1}\left(-e^{-2 f} \cos \left(x_{3}\right) \frac{\partial^{2} L}{\partial x_{2} \partial x_{1}} d x_{2}\right. \\
& +2 e^{-2 f} \cos \left(x_{3}\right) \frac{\partial f}{\partial x_{2}} \frac{\partial L}{\partial x_{1}} d x_{2} \\
& +e^{-2 f} \sin \left(x_{3}\right) \frac{\partial^{2} L}{\partial x_{2}^{2}} d x_{2} \\
& \left.-2 e^{-2 f} \sin \left(x_{3}\right) \frac{\partial f}{\partial x_{2}} \frac{\partial L}{\partial x_{2}} d x_{2}\right) \\
& +Y^{2}\left(-e^{-2 f} \sin \left(x_{3}\right) \frac{\partial^{2} L}{\partial x_{2} \partial x_{1}} d x_{2}\right. \\
& +2 e^{-2 f} \sin \left(x_{3}\right) \frac{\partial f}{\partial x_{2}} \frac{\partial L}{\partial x_{1}} d x_{2} \\
& -e^{-2 f} \cos \left(x_{3}\right) \frac{\partial^{2} L}{\partial x_{2}^{2}} d x_{2} \\
& \left.+2 e^{-2 f} \cos \left(x_{3}\right) \frac{\partial f}{\partial x_{2}} \frac{\partial L}{\partial x_{2}} d x_{2}\right) \\
& +Y^{3}\left(-\frac{\partial^{2} L}{\partial x_{2} \partial x_{3}} d x_{2}+2 e^{-2 f} \frac{\partial f}{\partial x_{2}} \frac{\partial}{\partial x_{3}} d x_{2}\right) \\
& -\frac{\partial L}{\partial x_{2}} d x_{2}+X^{1}\left(e^{2 f} \cos \left(x_{3}\right) \frac{\partial^{2} L}{\partial x_{3} \partial y_{1}} d x_{3}\right. \\
& +2 e^{2 f} \cos \left(x_{3}\right) \frac{\partial f}{\partial x_{3}} \frac{\partial L}{\partial y_{1}} d x_{3}-e^{2 f} \sin \left(x_{3}\right) \frac{\partial L}{\partial y_{1}} d x_{3} \\
& +e^{2 f} \sin \left(x_{3}\right) \frac{\partial^{2} L}{\partial x_{3} \partial y_{2}} d x_{3} \\
& \begin{array}{l}
\left.+e^{2 f} \cos \left(x_{3}\right) \frac{\partial L}{\partial y_{2}} d x_{3}\right) \\
+X^{2}\left(-e^{2 f} \sin \left(x_{3}\right) \frac{\partial^{2} L}{\partial x_{3} \partial y_{1}} d x_{3}\right.
\end{array} \\
& -2 e^{2 f} \sin \left(x_{3}\right) \frac{\partial f}{\partial x_{3}} \frac{\partial L}{\partial y_{1}} d x_{3}-e^{2 f} \cos \left(x_{3}\right) \frac{\partial L}{\partial y_{1}} d x_{3} \\
& +e^{2 f} \cos \left(x_{3}\right) \frac{\partial^{2} L}{\partial x_{3} \partial y_{2}} d x_{3} \\
& +2 e^{2 f} \cos \left(x_{3}\right) \frac{\partial f}{\partial x_{3}} \frac{\partial L}{\partial y_{2}} d x_{3} \\
& \left.-e^{2 f} \sin \left(x_{3}\right) \frac{\partial L}{\partial y_{2}} d x_{3}\right)+X^{3}\left(e^{2 f} \frac{\partial^{2} L}{\partial x_{3} \partial y_{3}} d x_{3}\right. \\
& \left.+2 e^{2 f} \frac{\partial f}{\partial x_{3}} \frac{\partial L}{\partial y_{3}} d x_{3}\right) \\
& +Y^{1}\left(-e^{-2 f} \cos \left(x_{3}\right) \frac{\partial^{2} L}{\partial x_{3} \partial x_{1}} d x_{3}\right. \\
& +2 e^{-2 f} \cos \left(x_{3}\right) \frac{\partial f}{\partial x_{3}} \frac{\partial L}{\partial x_{1}} d x_{3} \\
& -e^{-2 f} \sin \left(x_{3}\right) \frac{\partial L}{\partial x_{1}} d x_{3}+e^{-2 f} \sin \left(x_{3}\right) \frac{\partial^{2} L}{\partial x_{3} \partial x_{2}} d x_{3} \\
& -2 e^{-2 f} \sin \left(x_{3}\right) \frac{\partial f}{\partial x_{3}} \frac{\partial L}{\partial x_{2}} d x_{3} \\
& \left.+e^{-2 f} \cos \left(x_{3}\right) \frac{\partial L}{\partial x_{2}} d x_{3}\right) \\
& +Y^{2}\left(-e^{-2 f} \sin \left(x_{3}\right) \frac{\partial^{2} L}{\partial x_{3} \partial x_{1}} d x_{3}\right. \\
& +2 e^{-2 f} \sin \left(x_{3}\right) \frac{\partial f}{\partial x_{3}} \frac{\partial L}{\partial x_{1}} d x_{3} \\
& -e^{-2 f} \cos \left(x_{3}\right) \frac{\partial L}{\partial x_{1}} d x_{3} \\
& -e^{-2 f} \cos \left(x_{3}\right) \frac{\partial^{2} L}{\partial x_{3} \partial x_{2}} d x_{3} \\
& +2 e^{-2 f} \cos \left(x_{3}\right) \frac{\partial f}{\partial x_{3}} \frac{\partial L}{\partial x_{2}} d x_{3} \\
& \left.+e^{-2 f} \sin \left(x_{3}\right) \frac{\partial L}{\partial x_{2}} d x_{3}\right)+Y^{3}\left(-\frac{\partial^{2} L}{\partial x_{3}^{2}} d x_{3}\right. \\
& \left.+2 e^{-2 f} \frac{\partial f}{\partial x_{3}} \frac{\partial}{\partial x_{3}} d x_{3}\right)-\frac{\partial L}{\partial x_{3}} d x_{3}
\end{aligned}
$$




$$
\begin{aligned}
& +X^{1}\left(e^{2 f} \cos \left(x_{3}\right) \frac{\partial^{2} L}{\partial y_{1}^{2}} d y_{1}\right. \\
& \left.+2 e^{2 f} \sin \left(x_{3}\right) \frac{\partial f}{\partial y_{2}} \frac{\partial L}{\partial y_{2}} d y_{2}\right) \\
& +2 e^{2 f} \cos \left(x_{3}\right) \frac{\partial f}{\partial y_{1}} \frac{\partial L}{\partial y_{1}} d y_{1} \\
& +X^{2}\left(-e^{2 f} \sin \left(x_{3}\right) \frac{\partial^{2} L}{\partial y_{2} \partial y_{1}} d y_{2}\right. \\
& +e^{2 f} \sin \left(x_{3}\right) \frac{\partial^{2} L}{\partial y_{1} \partial y_{2}} d y_{1} \\
& \left.+2 e^{2 f} \sin \left(x_{3}\right) \frac{\partial f}{\partial y_{1}} \frac{\partial L}{\partial y_{2}} d y_{1}\right) \\
& +X^{2}\left(-e^{2 f} \sin \left(x_{3}\right) \frac{\partial^{2} L}{\partial y_{1}^{2}} d y_{1}\right. \\
& -2 e^{2 f} \sin \left(x_{3}\right) \frac{\partial f}{\partial y_{1}} \frac{\partial L}{\partial y_{1}} d y_{1} \\
& +e^{2 f} \cos \left(x_{3}\right) \frac{\partial^{2} L}{\partial y_{1} \partial y_{2}} d y_{1} \\
& \left.+2 e^{2 f} \cos \left(x_{3}\right) \frac{\partial f}{y_{1}} \frac{\partial L}{\partial y_{2}} d y_{1}\right)+X^{3}\left(e^{2 f} \frac{\partial^{2} L}{\partial y_{1} \partial y_{3}} d y_{1}\right. \\
& \left.+2 e^{2 f} \frac{\partial f}{\partial y_{1}} \frac{\partial L}{\partial y_{3}} d y_{1}\right) \\
& +Y^{1}\left(-e^{-2 f} \cos \left(x_{3}\right) \frac{\partial^{2} L}{\partial y_{1} \partial x_{1}} d y_{1}\right. \\
& +2 e^{-2 f} \cos \left(x_{3}\right) \frac{\partial f}{\partial y_{1}} \frac{\partial L}{\partial x_{1}} d y_{1} \\
& +e^{-2 f} \sin \left(x_{3}\right) \frac{\partial^{2} L}{\partial y_{1} \partial x_{2}} d y_{1} \\
& \left.-2 e^{-2 f} \sin \left(x_{3}\right) \frac{\partial f}{\partial y_{1}} \frac{\partial L}{\partial x_{2}} d y_{1}\right) \\
& +Y^{2}\left(-e^{-2 f} \sin \left(x_{3}\right) \frac{\partial^{2} L}{\partial y_{1} \partial x_{1}} d y_{1}\right. \\
& +2 e^{-2 f} \sin \left(x_{3}\right) \frac{\partial f}{\partial y_{1}} \frac{\partial L}{\partial x_{1}} d y_{1} \\
& -e^{-2 f} \cos \left(x_{3}\right) \frac{\partial^{2} L}{\partial y_{1} \partial x_{2}} d y_{1} \\
& \left.+2 e^{-2 f} \cos \left(x_{3}\right) \frac{\partial f}{\partial y_{1}} \frac{\partial L}{\partial x_{2}} d y_{1}\right)+Y^{3}\left(-\frac{\partial^{2} L}{\partial y_{1} \partial x_{3}} d y_{1}\right. \\
& \left.+2 e^{-2 f} \frac{\partial f}{\partial y_{1}} \frac{\partial}{\partial x_{3}} d y_{1}\right)-\frac{\partial L}{\partial y_{1}} d y_{1} \\
& +X^{1}\left(e^{2 f} \cos \left(x_{3}\right) \frac{\partial^{2} L}{\partial y_{2} \partial y_{1}} d y_{2}\right. \\
& +2 e^{2 f} \cos \left(x_{3}\right) \frac{\partial f}{\partial y_{2}} \frac{\partial L}{\partial y_{1}} d y_{2}+e^{2 f} \sin \left(x_{3}\right) \frac{\partial^{2} L}{\partial y_{2}^{2}} d y_{2} \\
& -2 e^{2 f} \sin \left(x_{3}\right) \frac{\partial f}{\partial y_{2}} \frac{\partial L}{\partial y_{1}} d y_{2}+e^{2 f} \cos \left(x_{3}\right) \frac{\partial^{2} L}{\partial y_{2}^{2}} d y_{2} \\
& \left.+2 e^{2 f} \cos \left(x_{3}\right) \frac{\partial f}{\partial y_{2}} \frac{\partial L}{\partial y_{2}} d y_{2}\right) \\
& +X^{3}\left(e^{2 f} \frac{\partial^{2} L}{\partial y_{2} \partial y_{3}} d y_{2}+2 e^{2 f} \frac{\partial f}{\partial y_{2}} \frac{\partial L}{\partial y_{3}} d y_{2}\right) \\
& +Y^{1}\left(-e^{-2 f} \cos \left(x_{3}\right) \frac{\partial^{2} L}{\partial y_{2} \partial x_{1}} d y_{2}\right. \\
& +2 e^{-2 f} \cos \left(x_{3}\right) \frac{\partial f}{\partial y_{2}} \frac{\partial L}{\partial x_{1}} d y_{2} \\
& +e^{-2 f} \sin \left(x_{3}\right) \frac{\partial^{2} L}{\partial y_{2} \partial x_{2}} d y_{2} \\
& \left.-2 e^{-2 f} \sin \left(x_{3}\right) \frac{\partial f}{\partial y_{2}} \frac{\partial L}{\partial x_{2}} d y_{2}\right) \\
& +Y^{2}\left(-e^{-2 f} \sin \left(x_{3}\right) \frac{\partial^{2} L}{\partial y_{2} \partial x_{1}} d y_{2}\right. \\
& +2 e^{-2 f} \sin \left(x_{3}\right) \frac{\partial f}{\partial y_{2}} \frac{\partial L}{\partial x_{1}} d y_{2} \\
& -e^{-2 f} \cos \left(x_{3}\right) \frac{\partial^{2} L}{\partial y_{2} \partial x_{2}} d y_{2} \\
& \left.+2 e^{-2 f} \cos \left(x_{3}\right) \frac{\partial f}{\partial y_{2}} \frac{\partial L}{\partial x_{2}} d y_{2}\right)+Y^{3}\left(-\frac{\partial^{2} L}{\partial y_{2} \partial x_{3}} d y_{2}\right. \\
& \left.+2 e^{-2 f} \frac{\partial f}{\partial y_{2}} \frac{\partial}{\partial x_{3}} d y_{2}\right)-\frac{\partial L}{\partial y_{2}} d y_{2} \\
& +X^{1}\left(e^{2 f} \cos \left(x_{3}\right) \frac{\partial^{2} L}{\partial y_{3} \partial y_{1}} d y_{3}\right. \\
& +2 e^{2 f} \cos \left(x_{3}\right) \frac{\partial f}{\partial y_{3}} \frac{\partial L}{\partial y_{1}} d y_{3} \\
& +e^{2 f} \sin \left(x_{3}\right) \frac{\partial^{2} L}{\partial y_{3} \partial y_{2}} d y_{3} \\
& \left.+2 e^{2 f} \sin \left(x_{3}\right) \frac{\partial f}{\partial y_{3}} \frac{\partial L}{\partial y_{2}} d y_{3}\right) \\
& +X^{2}\left(-e^{2 f} \sin \left(x_{3}\right) \frac{\partial^{2} L}{\partial y_{3} \partial y_{1}} d y_{3}\right. \\
& -2 e^{2 f} \sin \left(x_{3}\right) \frac{\partial f}{\partial y_{3}} \frac{\partial L}{\partial y_{1}} d y_{3}
\end{aligned}
$$




$$
\begin{aligned}
& +e^{2 f} \cos \left(x_{3}\right) \frac{\partial^{2} L}{\partial y_{3} \partial y_{2}} d y_{3} \\
& \left.+2 e^{2 f} \cos \left(x_{3}\right) \frac{\partial f}{\partial y_{3}} \frac{\partial L}{\partial y_{2}} d y_{3}\right)+X^{3}\left(e^{2 f} \frac{\partial^{2} L}{\partial y_{3}^{2}} d y_{3}\right. \\
& \left.+2 e^{2 f} \frac{\partial f}{\partial y_{3}} \frac{\partial L}{\partial y_{3}} d y_{3}\right) \\
& +Y^{1}\left(-e^{-2 f} \cos \left(x_{3}\right) \frac{\partial^{2} L}{\partial y_{3} \partial x_{1}} d y_{3}\right. \\
& +2 e^{-2 f} \cos \left(x_{3}\right) \frac{\partial f}{\partial y_{3}} \frac{\partial L}{\partial x_{1}} d y_{3} \\
& +e^{-2 f} \sin \left(x_{3}\right) \frac{\partial^{2} L}{\partial y_{3} \partial x_{2}} d y_{3} \\
& \left.-2 e^{-2 f} \sin \left(x_{3}\right) \frac{\partial f}{\partial y_{3}} \frac{\partial L}{\partial x_{2}} d y_{3}\right) \\
& +Y^{2}\left(-e^{-2 f} \sin \left(x_{3}\right) \frac{\partial^{2} L}{\partial y_{3} \partial x_{1}} d y_{3}\right. \\
& +2 e^{-2 f} \sin \left(x_{3}\right) \frac{\partial f}{\partial y_{3}} \frac{\partial L}{\partial x_{1}} d y_{3} \\
& -e^{-2 f} \cos \left(x_{3}\right) \frac{\partial^{2} L}{\partial y_{3} \partial x_{2}} d y_{3} \\
& \left.+2 e^{-2 f} \cos \left(x_{3}\right) \frac{\partial f}{\partial y_{3}} \frac{\partial L}{\partial x_{2}} d y_{3}\right)+Y^{3}\left(-\frac{\partial^{2} L}{\partial y_{3} \partial x_{3}} d y_{3}\right. \\
& \left.+2 e^{-2 f} \frac{\partial f}{\partial y_{3}} \frac{\partial}{\partial x_{3}} d y_{3}\right)-\frac{\partial L}{\partial y_{3}} d y_{3} .
\end{aligned}
$$

Using (11), we get first equations as follows:

$$
\begin{gathered}
X^{1}\left[-e^{2 f} \cos \left(x_{3}\right) \frac{\partial^{2} L}{\partial x_{1} \partial y_{1}} d x_{1}\right. \\
-e^{2 f} 2 \frac{\partial f}{\partial x_{1}} \cos \left(x_{3}\right) \frac{\partial L}{\partial y_{1}} d x_{1} \\
-e^{2 f} \sin \left(x_{3}\right) \frac{\partial^{2} L}{\partial x_{1} \partial y_{2}} d x_{1} \\
\left.-e^{2 f} 2 \frac{\partial f}{\partial x_{1}} \sin \left(x_{3}\right) \frac{\partial L}{\partial y_{2}} d x_{1}\right] \\
+X^{2}\left[-e^{2 f} \cos \left(x_{3}\right) \frac{\partial^{2} L}{\partial x_{2} \partial y_{1}} d x_{1}\right. \\
-e^{2 f} 2 \frac{\partial f}{\partial x_{2}} \cos \left(x_{3}\right) \frac{\partial L}{\partial y_{1}} d x_{1} \\
-e^{2 f} \sin \left(x_{3}\right) \frac{\partial^{2} L}{\partial x_{2} \partial y_{2}} d x_{1}
\end{gathered}
$$

$$
\begin{aligned}
& \left.-e^{2 f} 2 \frac{\partial f}{\partial x_{2}} \sin \left(x_{3}\right) \frac{\partial L}{\partial y_{2}} d x_{1}\right] \\
& +X^{3}\left[-e^{2 f} \cos \left(x_{3}\right) \frac{\partial^{2} L}{\partial x_{3} \partial y_{1}} d x_{1}\right. \\
& -e^{2 f} 2 \frac{\partial f}{\partial x_{3}} \cos \left(x_{3}\right) \frac{\partial L}{\partial y_{1}} d x_{1} \\
& -e^{2 f} \sin \left(x_{3}\right) \frac{\partial^{2} L}{\partial x_{3} \partial y_{2}} d x_{1} \\
& \left.-e^{2 f} 2 \frac{\partial f}{\partial x_{3}} \sin \left(x_{3}\right) \frac{\partial L}{\partial y_{2}} d x_{1}\right] \\
& +Y^{1}\left[-e^{2 f} \cos \left(x_{3}\right) \frac{\partial \partial^{2} L}{\partial y_{1}^{2}} d x_{1}\right. \\
& +Y^{2}\left[-e^{2 f} \cos \left(x_{3}\right) \frac{\partial y^{2} L}{\partial y_{2} \partial y_{1}} d x_{1}\right. \\
& \left.-e^{2 f} 2 \frac{\partial f}{\partial y_{1}} \sin \left(x_{3}\right) \frac{\partial L}{\partial y_{2}} d x_{1}\right] \\
& -e^{2 f} \sin \left(x_{3}\right) \frac{\partial f}{\partial y_{1}} \cos \left(x_{3}\right) \frac{\partial y_{2}}{\partial y_{1}} d x_{1} \\
& -e_{1}
\end{aligned}
$$$$
+Y^{3}\left[-e^{2 f} \cos \left(x_{3}\right) \frac{\partial^{2} L}{\partial y_{3} \partial y_{1}} d x_{1}\right.
$$$$
-e^{2 f} 2 \frac{\partial f}{\partial y_{3}} \cos \left(x_{3}\right) \frac{\partial L}{\partial y_{1}} d x_{1}
$$$$
-e^{2 f} \sin \left(x_{3}\right) \frac{\partial^{2} L}{\partial y_{3} \partial y_{2}} d x_{1}
$$$$
\left.-e^{2 f} 2 \frac{\partial f}{\partial y_{3}} \sin \left(x_{3}\right) \frac{\partial L}{\partial y_{2}} d x_{1}\right]=-\frac{\partial L}{\partial x_{1}} d x_{1} \text {. }
$$

\section{From here}

$$
\begin{aligned}
& -\cos \left(x_{3}\right) V\left(e^{2 f} \frac{\partial L}{\partial y_{1}}\right)-\sin \left(x_{3}\right) V\left(e^{2 f} \frac{\partial L}{\partial y_{2}}\right)+\frac{\partial L}{\partial x_{1}} \\
& =0 .
\end{aligned}
$$


If we think of the curve $\alpha$, for all equations, as an integral curve of $V$, that is, $V(\alpha)=(\partial / \partial t)(\alpha)$, we find the following equations:

$$
\begin{aligned}
& (P D E 1) \quad-\cos \left(x_{3}\right) \frac{\partial}{\partial t}\left(e^{2 f} \frac{\partial L}{\partial y_{1}}\right) \\
& -\sin \left(x_{3}\right) \frac{\partial}{\partial t}\left(e^{2 f} \frac{\partial L}{\partial y_{2}}\right)+\frac{\partial L}{\partial x_{1}}=0, \\
& (P D E 2) \quad \sin \left(x_{3}\right) \frac{\partial}{\partial t}\left(e^{2 f} \frac{\partial L}{\partial y_{1}}\right) \\
& -\cos \left(x_{3}\right) \frac{\partial}{\partial t}\left(e^{2 f} \frac{\partial L}{\partial y_{2}}\right)+\frac{\partial L}{\partial x_{2}}=0, \\
& (\text { PDE3 })-\frac{\partial}{\partial t}\left(e^{2 f} \frac{\partial L}{\partial y_{3}}\right)+\frac{\partial L}{\partial x_{3}}=0, \\
& (P D E 4) \quad \cos \left(x_{3}\right) \frac{\partial}{\partial t}\left(e^{-2 f} \frac{\partial L}{\partial x_{1}}\right) \\
& -\sin \left(x_{3}\right) \frac{\partial}{\partial t}\left(e^{-2 f} \frac{\partial L}{\partial x_{2}}\right)+\frac{\partial L}{\partial y_{1}}=0, \\
& (P D E 5) \quad \sin \left(x_{3}\right) \frac{\partial}{\partial t}\left(e^{-2 f} \frac{\partial L}{\partial x_{1}}\right) \\
& +\cos \left(x_{3}\right) \frac{\partial}{\partial t}\left(e^{-2 f} \frac{\partial L}{\partial x_{2}}\right)+\frac{\partial L}{\partial y_{2}}=0 \\
& \text { (PDE6) } \frac{\partial}{\partial t}\left(e^{-2 f} \frac{\partial L}{\partial x_{3}}\right)+\frac{\partial L}{\partial y_{3}}=0,
\end{aligned}
$$

such that the differential equations (21) are named conformal Euler-Lagrange equations on conformally flat manifold which is shown in the form of $(T M, g, \nabla, J)$. Also, therefore, the triple $\left(T M, \Phi_{L}, V\right)$ is called a conformal-Lagrangian mechanical system on $(T M, g, \nabla, J)$.

\section{Weyl-Euler-Lagrangian Equations for Conservative Dynamical Systems}

Proposition 26. We choose $F=\mathbf{i}_{V}, g=\Phi_{L}$, and $\lambda=2 f$ at (11) and, by considering (4), we can write Weyl-Lagrangian dynamic equation as follows:

$$
\mathbf{i}_{V}\left(e^{2 f} \Phi_{L}\right)=\mathbf{i}_{V}\left(\Phi_{L}\right)-d(2 f)
$$

The second part (11), according to the law of conservation of energy [32], will not change for conservative dynamical systems and $\mathbf{i}_{V}\left(\Phi_{L}\right)=\Phi_{L}(V)$,

$$
\begin{aligned}
\Phi_{L}(V)-2 d f & =d E_{L}, \\
\Phi_{L}(V) & =d E_{L}+2 d f=d\left(E_{L}+2 f\right) .
\end{aligned}
$$

From (21) above $L \rightarrow L+2 f$. So, we can write

$$
\begin{aligned}
& (\text { PDE7 })-\cos \left(x_{3}\right) \frac{\partial}{\partial t}\left(e^{2 f} \frac{\partial(L+2 f)}{\partial y_{1}}\right) \\
& -\sin \left(x_{3}\right) \frac{\partial}{\partial t}\left(e^{2 f} \frac{\partial(L+2 f)}{\partial y_{2}}\right) \\
& +\frac{\partial(L+2 f)}{\partial x_{1}}=0 \\
& (P D E 8) \quad \sin \left(x_{3}\right) \frac{\partial}{\partial t}\left(e^{2 f} \frac{\partial(L+2 f)}{\partial y_{1}}\right) \\
& -\cos \left(x_{3}\right) \frac{\partial}{\partial t}\left(e^{2 f} \frac{\partial(L+2 f)}{\partial y_{2}}\right) \\
& +\frac{\partial(L+2 f)}{\partial x_{2}}=0 \\
& \left(\text { PDE9) }-\frac{\partial}{\partial t}\left(e^{2 f} \frac{\partial(L+2 f)}{\partial y_{3}}\right)+\frac{\partial(L+2 f)}{\partial x_{3}}=0\right. \text {, } \\
& (P D E 10) \quad \cos \left(x_{3}\right) \frac{\partial}{\partial t}\left(e^{-2 f} \frac{\partial(L+2 f)}{\partial x_{1}}\right) \\
& -\sin \left(x_{3}\right) \frac{\partial}{\partial t}\left(e^{-2 f} \frac{\partial(L+2 f)}{\partial x_{2}}\right) \\
& +\frac{\partial(L+2 f)}{\partial y_{1}}=0 \\
& (P D E 11) \quad \sin \left(x_{3}\right) \frac{\partial}{\partial t}\left(e^{-2 f} \frac{\partial(L+2 f)}{\partial x_{1}}\right) \\
& +\cos \left(x_{3}\right) \frac{\partial}{\partial t}\left(e^{-2 f} \frac{\partial(L+2 f)}{\partial x_{2}}\right) \\
& +\frac{\partial(L+2 f)}{\partial y_{2}}=0 \\
& \text { (PDE12) } \frac{\partial}{\partial t}\left(e^{-2 f} \frac{\partial(L+2 f)}{\partial x_{3}}\right)+\frac{\partial(L+2 f)}{\partial y_{3}}=0,
\end{aligned}
$$

and these differential equations (24) are named Weyl-EulerLagrange equations for conservative dynamical systems which are constructed on conformally flat manifold (TM, $g, \nabla, J, F)$ and therefore the triple $\left(T M, \Phi_{L}, V\right)$ is called a WeylLagrangian mechanical system.

\section{Equations Solving with Computer}

The equations systems (21) and (24) have been solved by using the symbolic Algebra software and implicit solution is below:

$$
\begin{aligned}
& L\left(x_{1}, x_{2}, x_{3}, y_{1}, y_{2}, y_{3}, t\right) \\
& \quad=\exp (-i * t) * F_{1}\left(y_{3}-i * x_{3}\right)+F_{2}(t) \\
& \quad+\exp (t * i) * F_{3}\left(y_{3}+x_{3} * i\right) \text { for } f=0 .
\end{aligned}
$$




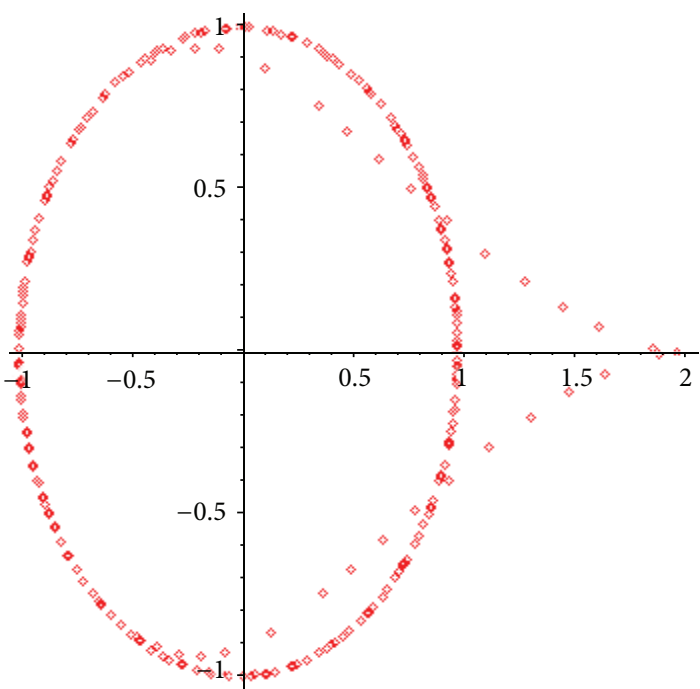

(a)

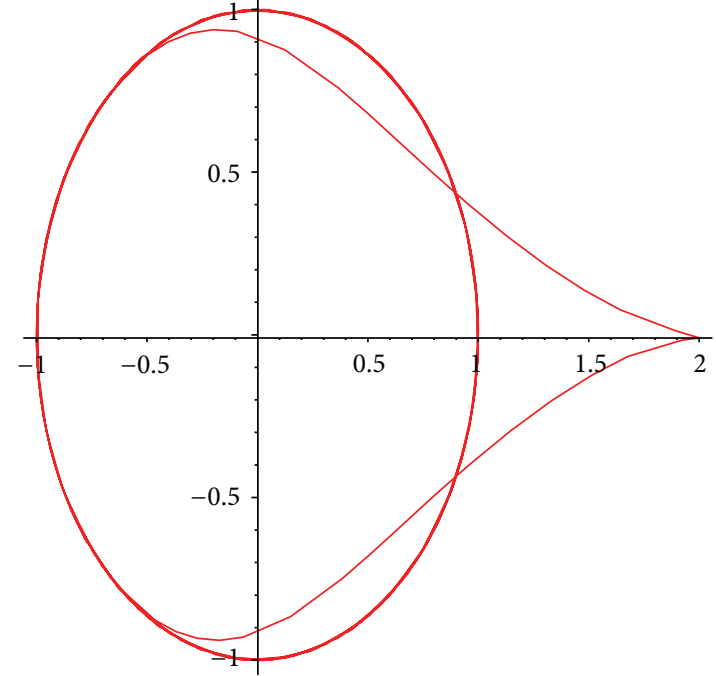

(b)

Figure 1

It is well known that an electromagnetic field is a physical field produced by electrically charged objects. The movement of objects in electrical, magnetic, and gravitational fields force is very important. For instance, on a weather map, the surface wind velocity is defined by assigning a vector to each point on a map. So, each vector represents the speed and direction of the movement of air at that point.

The location of each object in space is represented by three dimensions in physical space. The dimensions, which are represented by higher dimensions, are time, position, mass, and so forth. The number of dimensions of (25) will be reduced to three and behind the graphics will be drawn. First, implicit function at (25) will be selected as special. After the figure of (25) has been drawn for the route of the movement of objects in the electromagnetic field.

Example 27. Consider

$$
L\left(x_{1}, x_{2}, x_{3}, y_{1}, y_{2}, y_{3}, t\right)=\exp (-i * t)+\exp (t * i) * t-t^{2},
$$

(see Figure 1).

\section{Discussion}

A classical field theory explains the study of how one or more physical fields interact with matter which is used in quantum and classical mechanics of physics branches. In this study, the Euler-Lagrange mechanical equations (21) and (24) derived on a generalized on flat manifolds may be suggested to deal with problems in electrical, magnetic, and gravitational fields force for the path of movement (26) of defined space moving objects [24].

\section{Conflict of Interests}

The author declares that there is no conflict of interests regarding the publication of this paper.

\section{Acknowledgment}

This work was supported by the agency BAP of Pamukkale University.

\section{References}

[1] W. K. Liu and S. Jun, Computational Nanomechanics of Materials, American Scientific Publishers, Stevenson Ranch, Calif, USA, 2005.

[2] M. Tekkoyun and Y. Yayli, "Mechanical systems on generalizedquaternionic Kähler manifolds," International Journal of Geometric Methods in Modern Physics, vol. 8, no. 7, pp. 1419-1431, 2011.

[3] D. B. Fogel, Epistemology of a theory of everything: Weyl, Einstein, and the unification of physics [Ph.D. thesis], Graduate School of the University of Notre Dame, Notre Dame, Ind, USA, 2008.

[4] Z. Kasap and M. Tekkoyun, "Mechanical systems on almost para/pseudo-Kähler-Weyl manifolds," International Journal of Geometric Methods in Modern Physics, vol. 10, no. 5, Article ID 1350008, 8 pages, 2013.

[5] Z. Kasap, "Weyl-mechanical systems on tangent manifolds of constant $W$-sectional curvature," International Journal of Geometric Methods in Modern Physics, vol. 10, no. 10, Article ID 1350053, pp. 1-13, 2013.

[6] M. Kapovich, "Flat conformal structures on 3-manifolds, I: uniformization of closed seifert manifolds," Journal of Differential Geometry, vol. 38, no. 1, pp. 191-215, 1993.

[7] F. Schwartz, "A volumetric Penrose inequality for conformally flat manifolds," Annales Henri Poincaré, vol. 12, no. 1, pp. 67-76, 2011.

[8] R. S. Kulkarni, "Conformally flat manifolds," Proceedings of the National Academy of Sciences of the United States of America, vol. 69, pp. 2675-2676, 1972.

[9] I. G. Dotti and R. J. Miatello, "On the cohomology ring of flat manifolds with a special structure," Revista De La Uni On Matematica Argentina, vol. 46, no. 2, pp. 133-147, 2005. 
[10] A. Szczepanski, Kähler at Manifolds of Low Dimensions, Institut des Hautes Études Scientifiques, Bures-sur-Yvette, France, 2005.

[11] R. Bartnik, "The mass of an asymptotically flat manifold," Communications on Pure and Applied Mathematics, vol. 39, no. 5, pp. 661-693, 1986.

[12] M. D. M. González, "Singular sets of a class of locally conformally flat manifolds," Duke Mathematical Journal, vol. 129, no. 3, pp. 551-572, 2005.

[13] S. Akbulut and M. Kalafat, "A class of locally conformally flat 4-manifolds," New York Journal of Mathematics, vol. 18, pp. 733$763,2012$.

[14] S.-H. Zhu, "The classification of complete locally conformally flat manifolds of nonnegative Ricci curvature," Pacific Journal of Mathematics, vol. 163, no. 1, pp. 189-199, 1994.

[15] H. M. Abood, "Almost Hermitian manifold with flat Bochner tensor," European Journal of Pure and Applied Mathematics, vol. 3, no. 4, pp. 730-736, 2010.

[16] K. Olszak and Z. Olszak, "On 4-dimensional, conformally flat, almost $\varepsilon$-Kählerian manifolds," Journal of Geometry and Physics, vol. 62, no. 5, pp. 1108-1113, 2012.

[17] S. Upadhyay, "A bounding question for almost flat manifolds," Transactions of the American Mathematical Society, vol. 353, no. 3, pp. 963-972, 2001.

[18] 2015, http://mathworld.wolfram.com/FlatManifold.html.

[19] 2015, http://en.wikipedia.org/wiki/Conformally_flat_manifold.

[20] D. McDu and D. Salamon, J-Holomorphic Curves and Quantum Cohomology, AMS, 1995.

[21] 2015, http://en.wikipedia.org/wiki/Conformal_class.

[22] G. B. Folland, "Weyl manifolds," Journal of Differential Geometry, vol. 4, pp. 145-153, 1970.

[23] L. Kadosh, Topics in weyl geometry [Ph.D. thesis], University of California, Berkeley, Calif, USA, 1996.

[24] H. Weyl, Space-Time-Matter, Dover Publications, 1922, Translated from the 4th German edition by H. Brose, Methuen, London, UK; Dover Publications, New York, NY, USA, 1952.

[25] P. Gilkey and S. Nikčević, "Kähler and para-Kähler curvature Weyl manifolds," http://arxiv.org/abs/1011.4844.

[26] H. Pedersen, Y. S. Poon, and A. Swann, "The Einstein-Weyl equations in complex and quaternionic geometry," Differential Geometry and Its Applications, vol. 3, no. 4, pp. 309-321, 1993.

[27] P. Gilkey and S. Nikcevic, "Kähler-Weyl manifolds of dimension 4," http://arxiv.org/abs/1109.4532.

[28] M. Brozos-Vázquez, P. Gilkey, and E. Merino, "Geometric realizations of Kaehler and of para-Kaehler curvature models," International Journal of Geometric Methods in Modern Physics, vol. 7, no. 3, pp. 505-515, 2010.

[29] 2015, http://en.wikipedia.org/wiki/Weyl_tensor.

[30] R. Miron, D. Hrimiuc, H. Shimada, and S. V. Sabau, The Geometry of Hamilton and Lagrange Spaces, Kluwer Academic Publishers, 2002.

[31] J. Klein, "Espaces variationnels et mécanique," Annales de l'Institut Fourier, vol. 12, pp. 1-124, 1962.

[32] M. de Leon and P. R. Rodrigues, Methods of Differential Geometry in Analytical Mechanics, North-Holland, Elsevier, Amsterdam, The Netherlands, 1989.

[33] R. Abraham, J. E. Marsden, and T. Ratiu, Manifolds, Tensor Analysis and Applications, Springer, New York, NY, USA, 2001. 


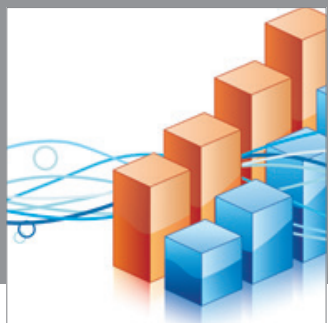

Advances in

Operations Research

mansans

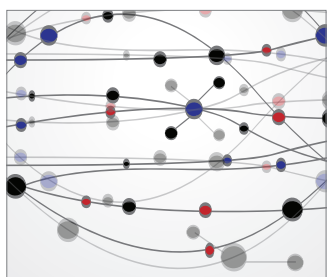

The Scientific World Journal
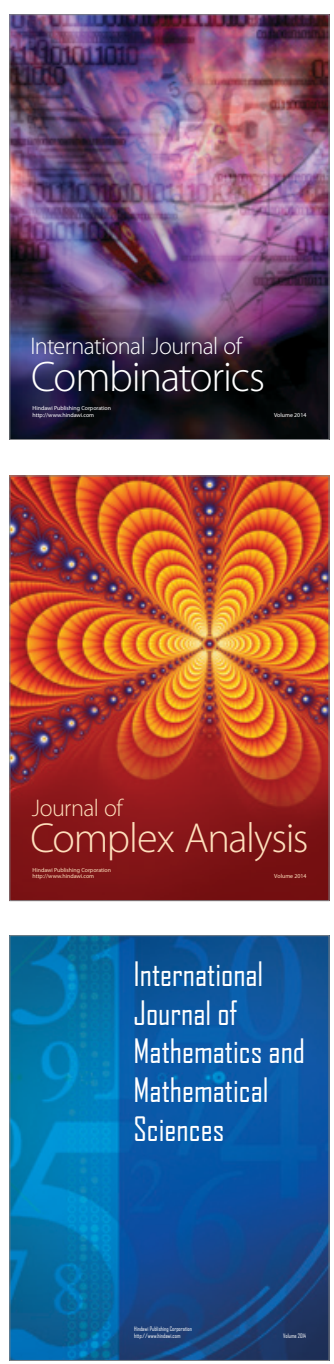
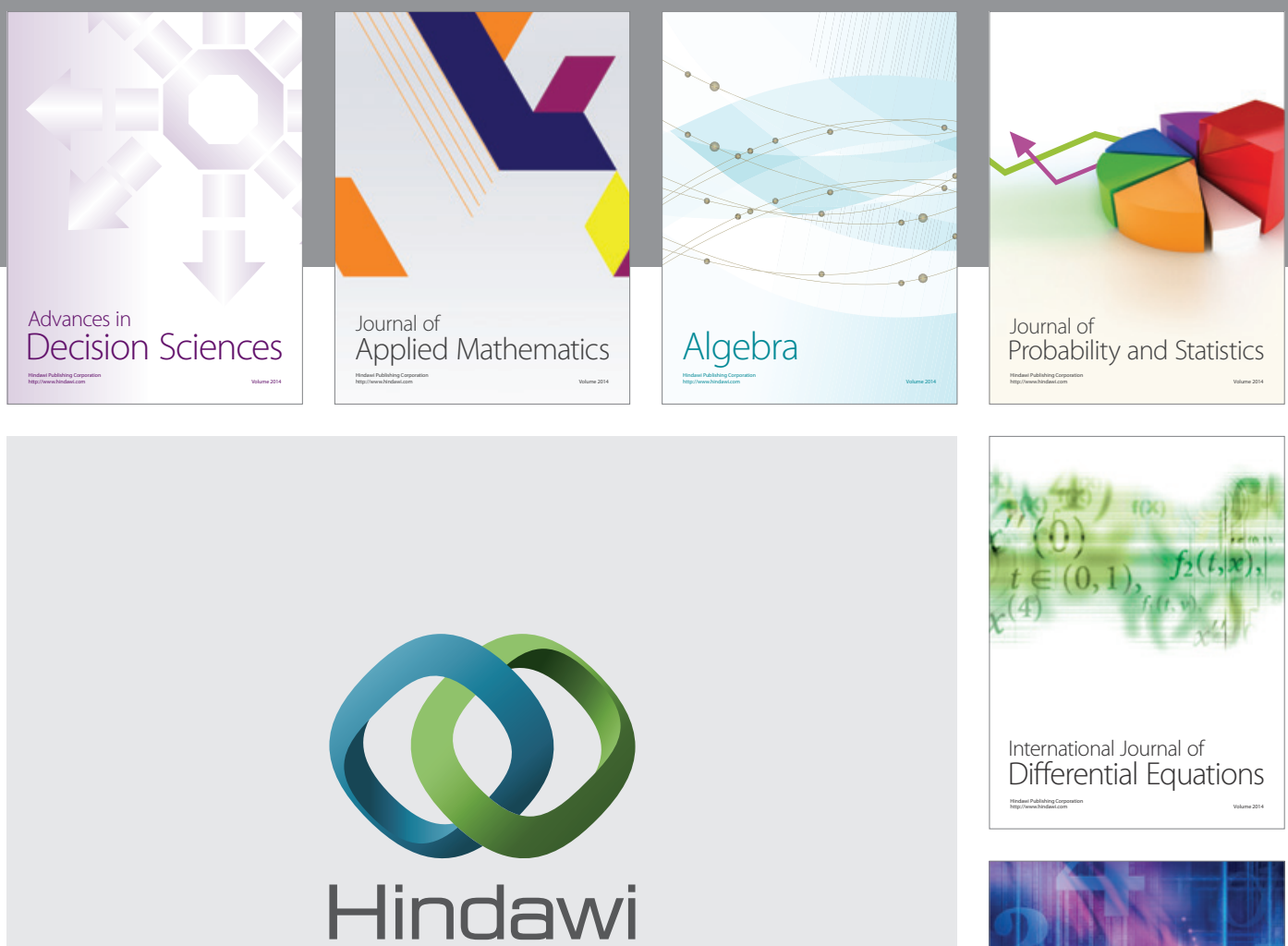

Submit your manuscripts at http://www.hindawi.com
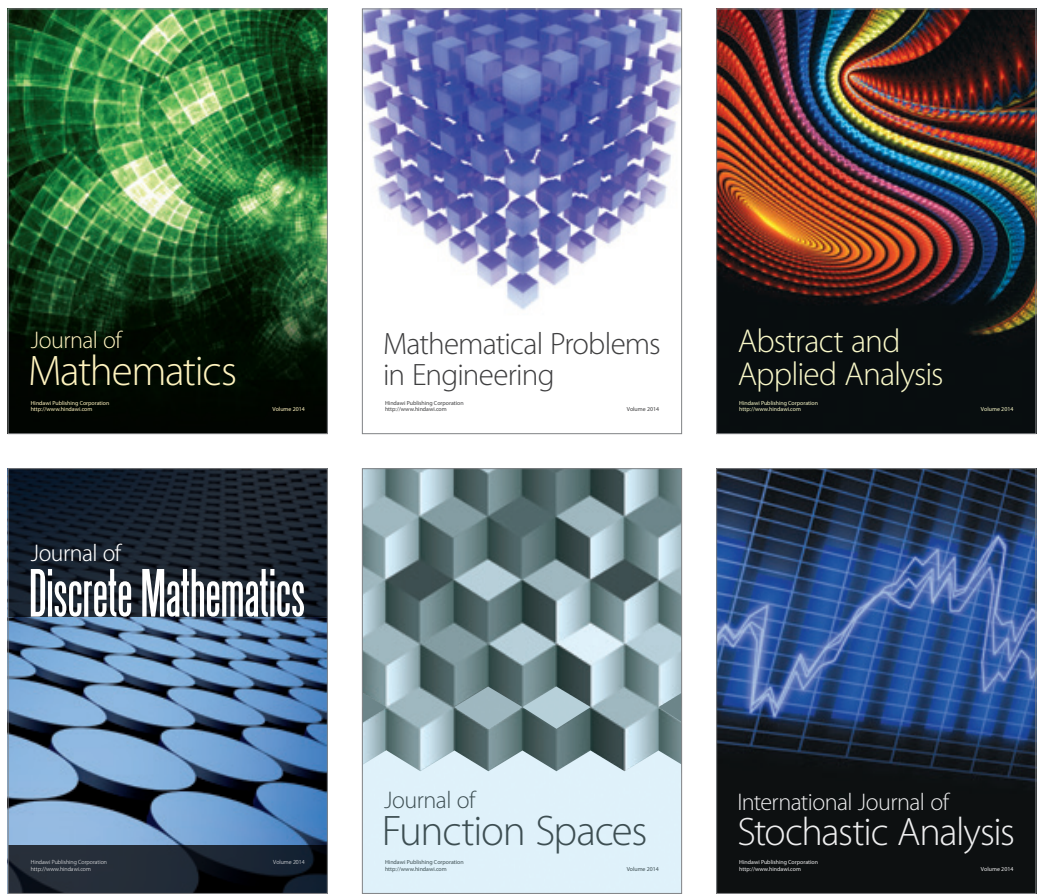

Journal of

Function Spaces

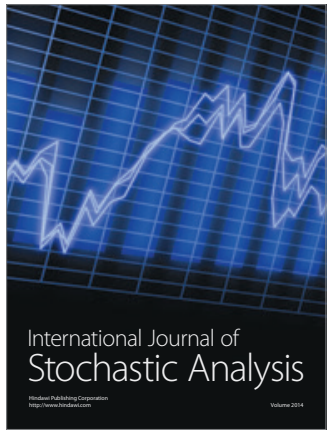

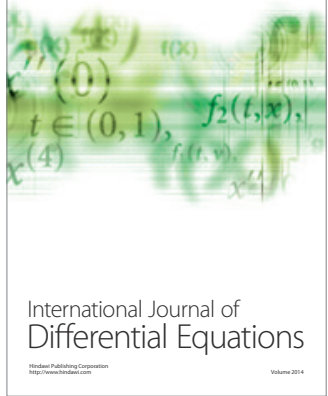
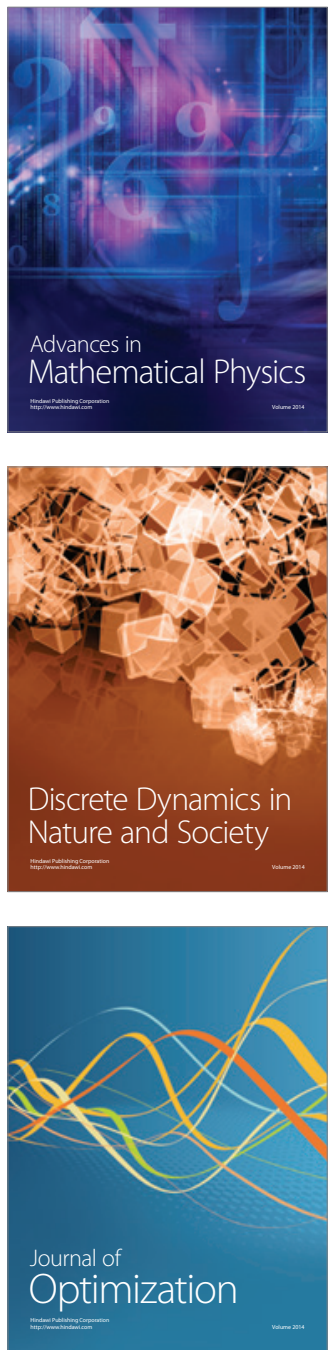